\title{
The Shorter the Better? An Inverted U-Shape Relationship between Service Duration and Value Judgment in Efficiency- Focused Services
}

\author{
Liang-Chu Ho \\ Doctoral Candidate, Institute of Education, \\ National Sun Yat-sen University, Taiwan \\ Wen-Bin Chiou \\ Professor, Institute of Education, \\ National Sun Yat-sen University, Taiwan
}

\begin{abstract}
Consumers tend to evaluate services based on the time required to perform such services, when the value of the latter positively correlates with efficiency. This study investigated how consumers use the efficiency heuristic, referring to the tendency of consumers to infer the value of a service from the perceived efficiency with which it is performed. We studied 81 participants who had experienced car problems. The relationship between time devoted to a given service and judgments about its value showed a quadratic trend, with an inverted U-shape, in regard to a service in which value was related to efficiency. Specifically, participants judged services to be more valuable when they involved relatively moderate amounts of time to complete, compared to when they involved comparatively shorter or longer periods of time. The current research suggests that when a service was judged in terms of efficiency, consumers seemed to apply the efficiency heuristic not only to the time required for service performance, but also to labor costs.
\end{abstract}

Keywords: Efficiency, Effort, Heuristic, Service duration, Value judgment

\section{INTRODUCTION}

Time represents one salient dimension in the provision of service to consumers. In many service situations, consumers have to spend more time to obtain more of the service. For example, participation in a physical fitness program requires sufficient time for the exercises to produce the desired results. However, the relationship between service duration and service value is negative in regard to many services, including locksmith issues, emergency rescues, apparatus maintenance, and travel planning. This relationship would be most salient when efficiency or speed of response correlates negatively with duration. Thus efficiency would represent a robust factor in judgments about the value of a particular service when time represents the major criterion for judgments about service quality (i.e., an efficiency-focused service). This article demonstrates that consumers tend to use the efficiency of a certain service (e.g., emergency car service) as a basis for evaluating that service. This tendency of consumers to infer the value of a service from its efficiency has been referred to as the "efficiency heuristic." In this study, considerations of labor cost appeared to be an important factor in the use of the efficiency heuristic by consumers in their service judgments.

\section{EFFICIENCY-FOCUSED SERVICES AND SERVICE JUDGMENTS}

For many services, efficiency carries value. That is, greater benefit can accrue to a service that takes less time than to a comparable service taking more time. For example, consumers are 
generally more satisfied when car damage (event $X$ ) requires less time than was estimated to repair (event $Y$ ). However, consumers also tend to believe that if repairs require less time than estimated (event $Y$ ), then rescue from situations involving a damaged vehicle (event $X$ ) follows a fixed schedule. This pattern reflects a more general tendency to treat conditional relations between events as bi-conditional.

More specifically, consumers who believe that one event implies another event (i.e., that $X$ implies $Y$ ) will also assume that the second implies the first (i.e., that $Y$ implies $X$ ) and, therefore, will infer $Y$ from $X$ (Wyer and Scrull [1]; Fiske and Taylor [2]; Yeung and Soman [3]). Other heuristics also depict the tendency to treat conditional relations between events as biconditional, including the representativeness heuristic (Kahneman and Tversky [4]; Tversky and Kahneman [5]). The representativeness heuristic refers to the tendency to believe that when members of a particular social category have certain attributes, then people who have these attributes belong to that category. For instance, when individuals believe that salesmen are generally sociable (i.e., that $X$ is $Y$ ), they then infer that people who are sociable are likely to be salesmen (i.e., that $Y$ is $X$ ), and that people who are not sociable are unlikely to be salesmen (i.e., that not- $Y$ is not- $X$ ). Therefore, we hypothesized that consumers would tend to use the efficiency heuristic in judging services. In particular, we predicted that consumers would infer the benefit deriving from a service, and hence the value of this service, from the efficiency with which the service was performed. Service of shorter duration would be judged more favorably than service of longer duration when value was determined by efficiency.

\section{Participants and Design}

\section{METHODOLOGY}

We determined the sample size for this study on the basis of the number of subjects necessary to achieve a specified power for a completely randomized factorial design (Kirk [6]). The significance level $(\alpha)$ was set at .05, the effect size (Cohen's $f$, Cohen [7]) was set at 0.4 , and the statistical power $(1-\beta)$ was set at 0.9 . Under these conditions, each cell had to contain 27 participants. Thus, we recruited 81 previous consumers of the service examined in this study and randomly assigned each person to one of three experimental conditions. The conditions were defined by manipulating the times involved in the subsequent delivery of the service into categories of short, medium, and long.

\section{Materials}

A "car rescue" scenario, in which consumers wanted a broken-down car repaired as quickly as possible, was employed in this experiment. The value of such a car rescue service presumably would correlate positively with the efficiency of the service, but negatively with the time required for that service. Participants in the short-duration group were asked to imagine that "you are driving on a highway to get to an important meeting. However, your car breaks down unexpectedly when you try to restart it at a rest area. You don't want to be late for this meeting because your client is too busy to reschedule. You call a service to fix your car as soon as possible. Your meeting schedule allows the technician only one and a half hours to fix the problem. The technician arrives 15 minutes later. It takes 30 minutes for the technician to finish the job, and he charges you $\$ 100$." Participants in the medium- and long-duration groups were asked to imagine identical scenarios, except that the technician spent 60 minutes repairing the car in the medium-duration condition and 90 minutes in the long-duration condition. The service durations were manipulated between subjects.

\section{Procedure}

Participants were informed that the present study was examining how people evaluate a certain service. After signing consent forms, they were presented with the hypothetical 
scenario. Following presentation of the scenario, participants were first asked to evaluate the value of the service (on a scale from one, meaning "not at all," to 11, meaning "very reasonable"), and asked to rate the perceived efficiency of the service (on a scale from one, meaning "not at all," to 11, meaning "very efficient"). Finally, all participants were asked to estimate "how much effort the service provider put into the service" (on a scale from one, meaning "none at all," to 11, meaning "very much"), and to rate the perceived duration of the service in the assigned condition (on a scale from one, meaning "very short," to 11, meaning "very long").

\section{Manipulation Check}

\section{RESULTS}

Perceptions about service duration and efficiency (Table 1) were subjected to separate trend analyses. The results indicated that the participants who imagined the long-duration condition perceived the service as taking the longest amount of time $(M=8.63)$; those who imagined the short-duration condition perceived the service as taking the shortest amount of time $(M=$ 3.22); and those who imagined the medium-duration condition perceived the service as taking an amount of time falling in between the other two conditions $(M=5.21$; the linear $F(1,78)=$ $\left.131.00, p<0.001 ; \eta^{2}=0.63\right)$. Participants in the short-duration condition judged the service as most efficient $(M=8.59)$; those in the long-duration condition judged the service as least efficient $(M=4.33)$; and those in the medium-duration condition judged the efficiency as between the other two ratings $\left(M=5.19\right.$; the linear $\left.F(1,78)=120.45, p<0.001 ; \eta^{2}=0.61\right)$.

Table 1: Descriptive statistics (means and standard deviations) of the measures

\begin{tabular}{lcccccc}
\hline & \multicolumn{9}{c}{ Duration } \\
\cline { 2 - 7 } Measure & \multicolumn{2}{c}{ Short } & \multicolumn{2}{c}{ Medium } & \multicolumn{2}{c}{ Long } \\
\cline { 2 - 7 } & $M$ & $S D$ & & $S D$ & $M$ & $S D$ \\
\hline Perceived duration & 3.22 & 1.37 & 5.21 & 1.82 & 8.63 & 1.96 \\
Perceived efficiency & 8.59 & 1.58 & 5.19 & 1.12 & 4.33 & 1.54 \\
Value judgments & 5.78 & 1.53 & 8.19 & 1.11 & 3.44 & 2.19 \\
Perceived effort & 3.78 & 1.37 & 5.79 & 1.82 & 6.37 & 1.96
\end{tabular}

Notes: Each experimental condition involved 27 participants. All measures were rated on an 11-point scale.

\section{Service Judgments}

An overall analysis of the data regarding judgments about service (Table 1 ) yielded a quadratic trend, with a U-shaped curve (the quadratic $F(1,78)=82.49, p<0.001 ; \eta^{2}=0.51$ ). The participants evaluated the service as more valuable when it was of medium duration $(M=$ 8.19), than when it was of shorter $(M=5.78)$ or longer $(M=3.44)$ duration. Supplementary analysis of the differences between judgments of efficiency and effort showed that, among the participants in the short-duration condition, perceived efficiency was significantly greater than perceived effort $(t=11.74, p<0.001)$; among participants in the long-duration condition, perceived efficiency was significantly lower than perceived effort $(t=-4.25, p<0.001)$; and among participants in the medium-duration condition, perceived efficiency and effort were comparable at a moderate level $(t=-1.36, p>0.05)$. 


\section{DISCUSSION}

The objective of this experiment was to examine whether consumers' service judgments were contingent on the length of time required to perform a given service. The present study manipulated the duration of service (short, medium, and long) in the context of a car rescue scenario and asked participants to evaluate the service in terms of its efficiency. The participants were expected to privilege efficiency as the framework for judging service because they were prompted to consider efficiency as a criterion in evaluation. Thus we hypothesized that participants would be more likely to use the efficiency heuristic and to judge the service as more valuable when its duration was shorter than when it was longer; that is, when the service was efficient rather than inefficient. However, the findings showed an interesting and surprising pattern that was inconsistent with these predictions. When participants judged a service in which the duration and the value correlated negatively (i.e., a car rescue), the service involving the shortest duration was not judged as the most valuable. Instead, participants judged the service involving a medium duration as the most valuable.

We tentatively explain this result as follows. Participants in the long-duration condition might have attributed the length of time required to complete the service to inefficiency rather than to greater effort (i.e., labor cost) on the part of the car service; this notion was in accord with the finding that perceived efficiency was apparently lower than perceived effort when the service took longer. By the same token, participants in the short-duration condition might have attributed the short service time to the simplicity and less effortful nature of the car repairs rather than to the technician's efficiency. If this were the case, the perceived effort involved in repairs involving short durations would be judged to be lower than the perceived efficiency in this condition, owing to assumptions that the technician expended less effort in the repair process. The data were congruent with such explanations. Furthermore, responses of those in the medium-duration condition indicated that moderate efficiency, accompanied by a comparable level of perceived effort, was associated with the most positive service judgments.

The finding of an inverted U-shaped relationship between service duration and service value suggests that consumers might have used an efficiency heuristic to compare between service duration and labor cost in judging a service that is valued for its efficiency. The matching hypothesis, referring to consumers' use of the efficiency heuristic, involves consideration of the compatible relationship between service duration and labor cost and is proposed as an explanation of the aforementioned findings. The most favorable judgments occurred when perceived efficiency and perceived effort were comparable. However, consumers in the shortduration condition also perceived that relatively little effort was required to complete the repairs. The effect of efficiency on judgments of value was undermined in the condition in which value was judged in relation to perceived effort. For the long-duration condition, the efficiency of a service became more robust than the effort it presumably involved because the particular service in question was valued in terms of its efficiency. When an efficiency-focused perspective was applied in the medium-duration condition, consumers responded with more favorable ratings of the service than they did in either the shorter or longer duration condition. The present findings support the matching hypothesis, which may explain and predict how the interaction of service duration and labor cost impacts the judgments made by consumers about service when that service is valued in terms of its efficiency.

\section{LIMITATIONS AND FUTURE DIRECTIONS}

The car rescue scenario was employed in this research; investigations into other kinds of services may also lend further support for the arguments presented in this article. Further, in the present study, we did not manipulate the price of a service and the perceived effort involved in that service. Although participants' price judgments were congruent with their 
value judgments, future studies should examine whether consumers' "willingness to pay" would be determined by the interaction of service duration and perceived effort, and would thereby support the matching hypothesis proposed in this article.

Future research should also consider factors that might mediate consumers' use of efficiency as a criterion for judging service. A fast pace is a salient feature of modern life (Hendry and Kinnison [8]; Kirkcaldy et al. [9]; Riley [10]). The pace of life may play a critical role in the goal of efficiency pursued in certain nations (Levine [11]). Levine and his colleagues conducted cross-cultural studies and identified five factors that determine the pace of life in a specific country or area (Levine [12]; Levine and Norenzayan [13]). They found that when a country was economically prosperous, highly industrialized, densely populated, cooler in climate, and placed greater value on individualism, the pace of life was faster. Therefore, future studies should utilize these factors (i.e., economic development, industrialization, population, climate, and individualism) in cross-cultural designs examining whether consumers from different countries or areas exhibit differential emphases on efficiency as a criterion for judgments about service.

Finally, according to Hsee et al. [14], it seems to be difficult for decision makers to evaluate an attribute when they do not have much knowledge about the effective range, a neutral reference point, and the value distribution of that attribute. The duration of service, as employed in the present study, precisely matches this description. Future research might examine whether the ability to evaluate a particular attribute of a specific service moderates the impact of the efficiency heuristic on judgments about service. More specifically, future research should address whether the time involved in the performance of a service that is highly amenable to evaluation (e.g., the cost of advertising or the fee charged by a lawyer) would undermine the use of an efficiency heuristic by consumers.

\section{MANAGERIAL IMPLICATIONS}

As discussed in this article, the efficiency heuristic produced a more pronounced effect on judgments about service when consumers evaluated the value of a service in relation to the time required to perform it. The inverted U-shaped relationship between service duration and service value suggests that marketing departments should understand that the judgments of consumers will be determined by the perceived efficiency and labor costs associated with a certain service. Hence, a promotional advantage might be obtained by framing an efficiencyfocused service in terms of optimal efficiency and labor cost. The results of this research provide alternative strategies for establishing competitive advantages by emphasizing efficiency in advertisements promoting services.

\section{ACKNOWLEDGEMENTS}

We would like to thanks the financial support received by the Lee Teng-hui Center for Governmental Studies from The Featured Areas Research Center Program within the framework of the Higher Education Sprout Project by the Ministry of Education (MOE) in Taiwan.

\section{References}

Wyer R. S., Scrull T. K. Memory and cognition in its social context. Hillsdale, NJ: Erlbaum, 1989.

Fiske S. T., Taylor, S. E. Social cognition. Hightstown, NJ: McGraw-Hill, 1991.

Yeung C. W., Soman D. The duration heuristic. Journal of Consumer Research 2007; 34: 315-326.

Kahneman D., Tversky A. Subjective probability: a judgment of representativeness. Cognitive Psychology 1971; 3: 430-454.

Tversky A., Kahneman D. Judgment under uncertainty: heuristics and biases. Science 1974; 185: 1124-1131. 
Kirk R. E. Experimental design: Procedures for the behavior science (4th ed.). Pacific Grove, CA: Brooks/Cole, 1995.

Cohen J. Statistical power analysis for the behavioral sciences. Hillsdale, NJ: Erlbaum, 1988.

Hendry A. P., Kinnison M. T. The pace of modern life: measuring rates of contemporary microevolution. Evolution 1999; 53: 1637-1653.

Kirkcaldy B., Furnham A., Levine R. Attitudinal and personality correlates of a nation's pace of life. Journal of Managerial Psychology 2001; 16: 20-34.

Riley J. C. The timing and pace of health transitions around the world. Population and Development Review 2005; 31: 741-764.

Levine R. The pace of life. American Scientist 1990; 78: 450-459.

Levine R. Geography of time: on tempo, culture and the pace of life. New York: Basic Books, 1997.

Levine R., Norenzayan A. The pace of life in 31 countries. Journal of Cross-Cultural Psychology 1999; 30: 178-205.

Hsee C. K., Blount S., Loewenstein G. F., Bazerman M. H. Preference reversals between joint and separate evaluations of options: a review and theoretical analysis. Psychological Bulletin 1999; 125: 576-590. 